\title{
Survival Rates for Breast Cancer in Iranian Patients: a Meta- Analysis
}

\author{
Mitra Rahimzadeh ${ }^{1}$, Mohamad Amin Pourhoseingholi², Behrooz Kavehie ${ }^{3 *}$
}

\begin{abstract}
Background: Breast cancer is the most common cancer among Iranian women. Since development of the disease in Iranian women occurs relatively early, the survival rate matters considerably. In different countries, survival of breast cancer patients varies considerably. Therefore, the one-year, three-year, five-year, and tenyear survival rates for breast cancer in Iran were assessed using a meta-analysis. Materials and Methods: This systematic review and meta-analysis was based on valid Iranian sources including SID, MAGIRAN and IRANMEDEX, along with reliable English databases, namely, PUBMED and SCOPUS. In domestic databases, a search was conducted based on key words of breast cancer and survival rate, and in international databases, with "breast cancer" and the equivalent of "neoplasm" of Mesh Word, "survival rate" and "Iran." Then all reviewed papers and theses which met the inclusion criteria were selected for investigation. To conduct the analysis STATA 11.2 software and random-effects models were used. Results: In 24 studies, 22,745 participants were included. The one-year, three-year, five-year and ten-year survival rates were $0.956,0.808,0.695$ and 0.559 , respectively. The minimum and maximum survival rates for 5 -years were 0.48 and 0.87 . The average age of the onset of the disease was 48.3. Conclusions: As in Iran, since the onset of the disease is at low age, in spite of the relatively high survival rate as compared to other cancers, prevention and screening programs at early age for early stage diagnosis seems necessary.
\end{abstract}

Keywords: Breast cancer - survival rate - meta-analysis - systematic review - Iran

Asian Pac J Cancer Prev, 17 (4), 2223-2227

\section{Introduction}

Breast cancer is a major public health problem throughout the world. This malignancy is the most common cancer in women, especially in the Eastern Mediterranean region (World health organization regional Office for the Eastern Mediterranean., 2006). 16\% of all cancers in Iran are connected to the breast cancer having ranked the first among Iranian women (Islamic Republic of Iran, Ministry of Health and Medical Education, Center for Disease Control \& Prevention. Noncommunicable Deputy, Cancer Office., 2009).

According to reports, in the United States one woman out of eight will be diagnosed with breast cancer (Miller et al., 2004). The risk of developing the disease in the lifetime is $12 \%$ and the chance of death because of the disease is 5 percent (Querzoli et al., 2006). Breast cancer is the fifth most fatal cancer after gastric cancer, leukemia, lung and bronchus, liver and bile ducts in Iranian women (Akbari., 2008) and its burden increasing recently (Sharifian et al., 2015). This cancer has caused $24.4 \%$ of all malignant tumors in Iran and is the most common cancer in Iranian women (Kolahdoozan et al., 2010). Beside the high prevalence of the breast cancer in Iran, acknowledging the fact that Iranian women, in comparison with developed countries, are diagnosed with the cancer at least a decade earlier (Taghavi et al., 2012), makes it doubly important.

Thus, knowledge of prognostic factors in breast cancer mortality risk can play an important role in the treatment and care of patients. The survival rate after diagnosis and treatment of cancer is one of the most important indicators used in the treatment and assessment procedures. Although there have been several studies to determine influencing factors on the survival of patients and to estimate the survival duration in patients suffering from breast cancer, studies on Iranian patients have created different results at different points (Harirchi et al., 2004; Moradi-Marjaneh et al., 2005; Salehi et al., 2013; Yaghmaei et al., 2007; Moetamed et al., 2004). The factors identified in the study in many cases are different from those in other studies and the impact rate of factors is also under discussion. Up to now, many other studies have been conducted to determine 


\section{Mitra Rahimzadeh et al}

the survival rate in breast cancer patients, but the topic is only discussed in small scale samples and in none of them, a general estimation of survival rate has been presented. The purpose of this study is to evaluate the survival rate of breast cancer patients in Iran to provide an estimation of the survival rate of breast cancer patients, using systematic review and meta-analysis method.

\section{Materials and Methods}

Data source: As this research is a systematic review and meta-analysis on survival in patients with breast cancer in Iran, the information required by the search with the key words "breast cancer" and "Survival" in E-Persian databases such as SID, MAGIRAN and IRANMEDEX including Persian articles and resources needed to collect the data. Moreover, to collect articles published in English, international databases, such as PUBMED and SCOPOUS with the keyword "Breast Cancer" and the equivalent of "neoplasm" of Mesh Word, "Survival" and "Iran" were used.

\section{Selection criteria}

All articles and dissertations examining the survival rate and the survival of women with breast cancer, without limitation by the year 2014 , were added to the study. Those studies on breast cancer which had been discussed but the survival rates were not reported or not adequate information, or to those data which access was not possible, were excluded. To avoid bias, the search was conducted independently by two researchers and the results of the repeated searches were omitted. Based on this strategy, 377 articles related to breast cancer were found. Out of this number, 38 articles because of being repetitive and 183 articles because of being irrelevant were eliminated. Out of the other 116 articles, having read the abstracts, 93 articles were excluded because of lack of information needed, repetitiveness of data used, checking the survival data of specific groups, were excluded. As a result, 23 articles and one thesis were included in metaanalysis procedure to be studied (Moradi-Marjaneh et al., 2005; Akbari et al., 2006; Vahdaninia et al., 2004; Fardmal et al., 2014; Hasanzadeh., 2014; Mokarian et al., 2013; Hagigat et al., 2013; Yaghmaei et al., 2008; Rajaeifard et al., 2006; Gohari et al., 2013; Mokhtari-Hesari et al., 2013; Babaei et al., 2005; Fouladi et al., 2011; Movahedi et al., 2012; Fallahzadeh et al., 2014; Akbari et al., 2008; Asadzadeh-Vostakolaei et al., 2012; Vostakolaei et al., 2012; Rezaianzadeh et al., 2009; Mousavi et al., 2008; Ziaei et al., 2013; Karimi et al., 2014; Heydari et al., 2009; Fazeli et al., 2014; Ghavam-Nasiri et al., 2005) (Figure 1).

\section{Data extraction}

All final articles, imported into the study process, provided by a pre-prepared check list, were arranged to extract the data. The check list was included the name of the first author, the sample size, location of the study, type of study, the average and age diagnostic of the patients, the survival rate of one, three, five, and ten-years, and the survival rate of 5-years, due to lack of information, stage of disease were excluded from the final check list.
Statistical Analysis:

In each study, the survival rates of one, three, five and ten year-old were considered as a binomial distribution and the variances were calculated based on the binomial distribution. To calculate the heterogeneity, among the studies, Q test and I2 index were used. According to these indexes, heterogeneity in the studies was approved. As a result, we used the random effect model to combine the results of selected studies. The data were analyzed using STATA 11.2 software and the meaningful level of 0.05 was set.

\section{Results}

There were 22745 patients in this study. Out of 24 analyses that met the inclusion criteria, all 24 studies had the 5-year survival rate. The minimum 5-year survival rate was estimated in Gavam Nasiri study with of 0.48 survival rate (Ghavam-Nasiri et al., 2005) and the maximum 5-year survival rate was estimated in Fazeli study with the survival rate of 0.87 (Fazeli et al., 2014). In the final analysis, the 5-year survival rate was 0.695 with the confidence interval of ( 0.657 and 0.734$)$ (Figure 2 ). Out of 16 studies with the survival rate of one year, with 11671 patients, the minimum 1-year survival rate was estimated in Yaghmaei study with of 0.869 survival

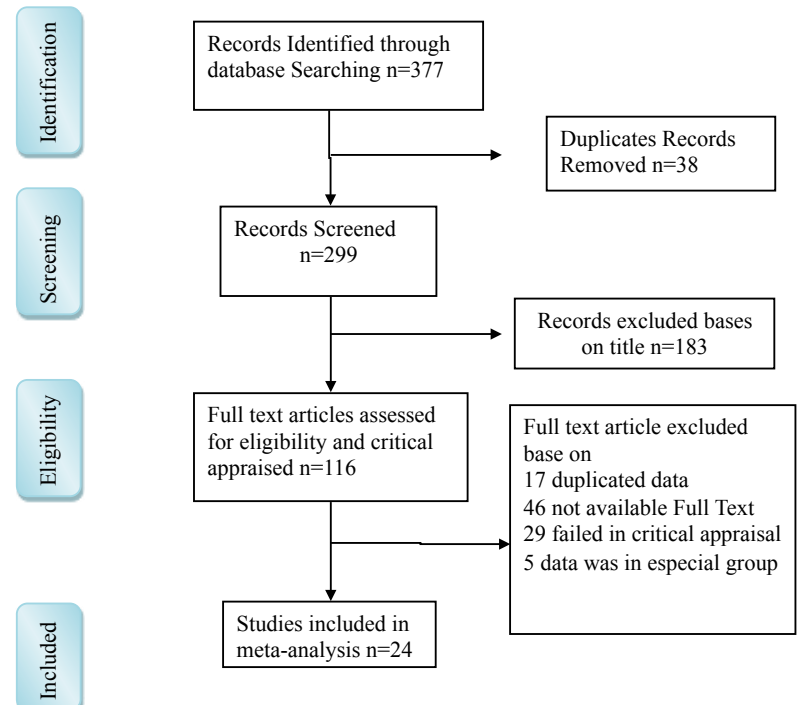

Figure 1. Selection Process for the Studies Included in the Systematic Review and Meta-Analysis

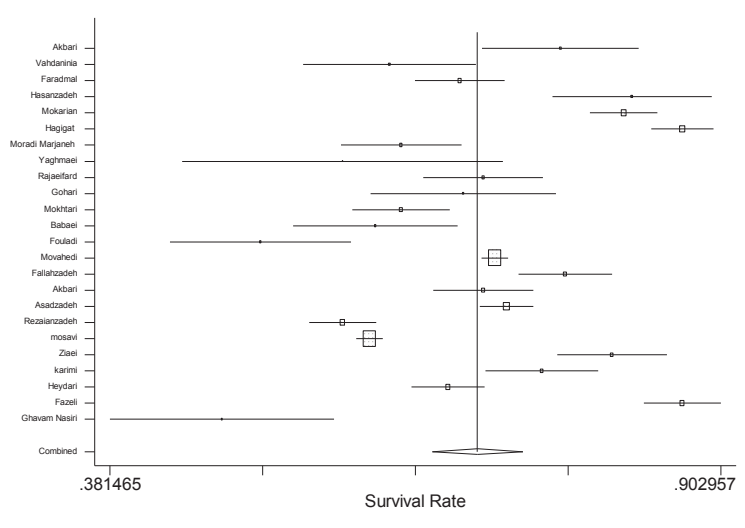

Figure 2. Five-Year Survival Rate in the Meta-Analysis 
Table 1. One, Three, Five and Ten Year Survival Rates According to the Meta-Analysis

\begin{tabular}{|c|c|c|c|c|c|c|}
\hline & Num Of study & Sample Size & Survival Rate & $95 \% \mathrm{CI}$ & minimum & maximum \\
\hline One year & 16 & 11671 & 0.956 & $0.943-970$ & 0.869 & 1 \\
\hline Three year & 18 & 12952 & 0.808 & $0.785-0.832$ & 0.62 & 0.92 \\
\hline Five year & 24 & 22745 & 0.695 & $0.657-0.734$ & 0.477 & 0.87 \\
\hline Ten year & 8 & 3159 & 0.559 & $0.433-0.685$ & 0.31 & 0.77 \\
\hline
\end{tabular}

Table 2. Survival Rates of Breast Cancer According to Papers Exclusion in Meta-analysis

\begin{tabular}{lccc}
\hline Study ommited & Survival Rate & \multicolumn{2}{c}{ [95\% Conf. Interval] } \\
\hline Akbari & 0.69231 & 0.65289 & 0.73173 \\
Vahdaninia & 0.69832 & 0.65892 & 0.73773 \\
Faradmal & 0.69586 & 0.65585 & 0.73588 \\
Hasanzadeh & 0.68984 & 0.65068 & 0.72900 \\
Mokarian & 0.68965 & 0.65115 & 0.72815 \\
Hagigat & 0.68777 & 0.65186 & 0.72368 \\
Moradi Marjaneh & 0.69811 & 0.65852 & 0.73770 \\
Yaghmaei & 0.69872 & 0.65961 & 0.73782 \\
Rajaeifard & 0.69501 & 0.65529 & 0.73473 \\
Gohari & 0.69574 & 0.65630 & 0.73518 \\
Mokhtari & 0.69817 & 0.65844 & 0.73789 \\
Babaei & 0.69886 & 0.65948 & 0.73825 \\
Fouladi & 0.70275 & 0.66379 & 0.74172 \\
Movahedi & 0.69364 & 0.64829 & 0.73899 \\
Fallahzadeh & 0.69185 & 0.65226 & 0.73145 \\
Akbari & 0.69498 & 0.65509 & 0.73486 \\
Asadzadeh & 0.69384 & 0.65285 & 0.73483 \\
Rezaianzadeh & 0.70071 & 0.66164 & 0.73979 \\
mosavi & 0.70038 & 0.66399 & 0.73676 \\
Ziaei & 0.69020 & 0.65101 & 0.72939 \\
karimi & 0.69280 & 0.65317 & 0.73244 \\
Heydari & 0.69628 & 0.65598 & 0.73659 \\
Fazeli & 0.68760 & 0.65028 & 0.72493 \\
Ghavam Nasiri & 0.70307 & 0.66448 & 0.74227 \\
Combined & 0.69528 & 0.65676 & 0.73381 \\
\hline & & &
\end{tabular}

rate (Yaghmaei et al., 2008) and the maximum 1-year survival rate was estimated in Hassanzadeh study with the survival rate of 1 (Hasanzadeh., 2014). The one-year survival rate was 0.956 with the confidence interval of (0.943 and 0.970) (Figure 3). Out of 18 studies with the 3- year survival rate, with 12952 patients, the minimum 3-year survival rate was estimated in Yaghmaei study with of 0.62 survival rate (Yaghmaei et al., 2008) and the maximum 3-year survival rate was estimated in Fazeli study with the survival rate of 0.92 (Fazeli et al., 2014). The three-year survival rate was 0.808 with the confidence interval of (0.785-0.832) (Figure 4). Only in 8 studies the 10-year survival rate was estimated with 3159 patients, the minimum survival rate of 10 -year was estimated in Faradmal with the lowest survival rate of 0.31 (Fardmal et al., 2014) and the highest survival rate of 10-year was estimated in Akbari with the survival rate of 0.77 (Akbari et al., 2008). In the final analysis, the ten-year survival rate was 0.559 with the confidence interval $(0.433$ and 0.685$)$ (Table 1) (Figure 5).

For further investigations, the studies according to their sample size were divided into three groups, namely, less than 500 participants, between 500 and 1000 participants, and more than 1,000 participants. In the first group, there were 15 studies with 3576 participants, the second group 5 studies with 3276 participants, and the

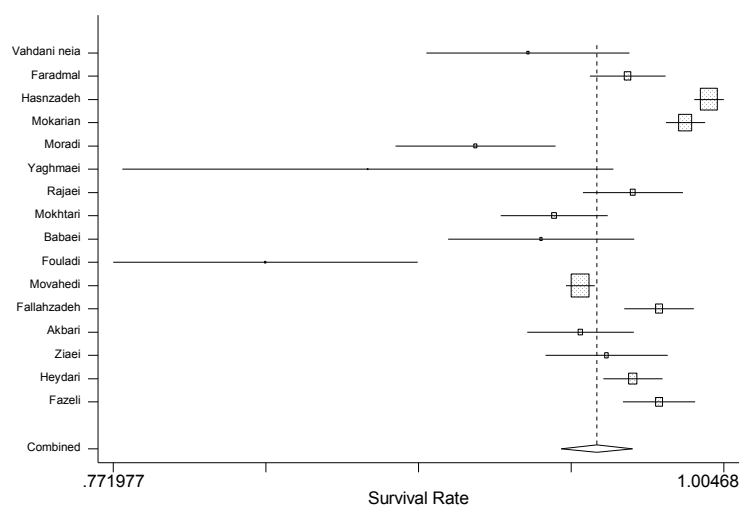

Figure 3. One-Year Survival Rate in the Meta-Analysis

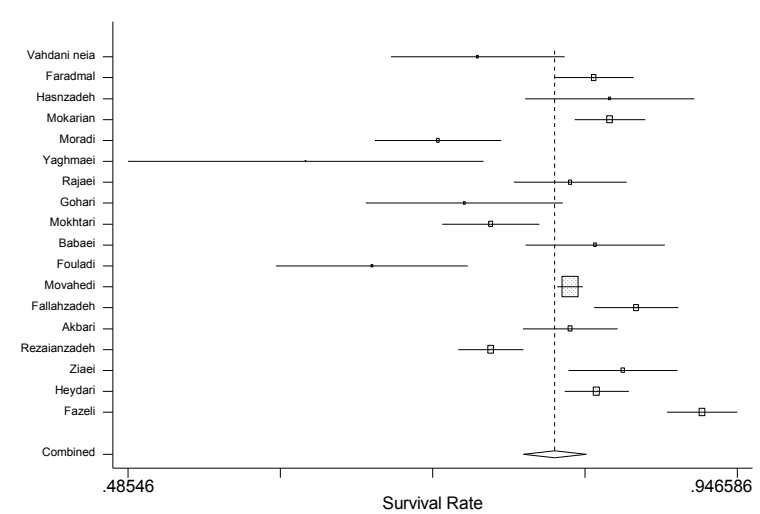

Figure 4. Three-Year Survival Rate in the MetaAnalysis

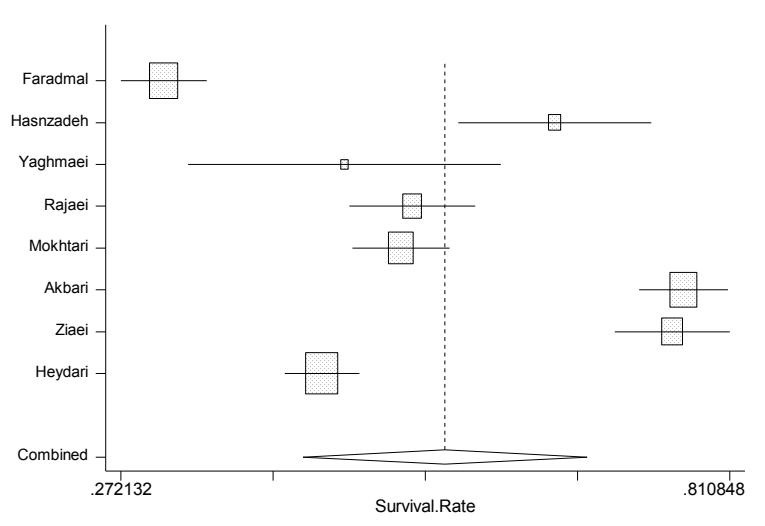

Figure 5. Ten-Year Survival Rate in the Meta-Analysis

third group 4 studies with 15893 participants.

In first group, the average 5-year survival rate was 0.692 with the confidence interval of (0.638 and 0.747). In second group, the average 5-year survival rate was 0.641 with the confidence interval of (0.735 and 0.829$)$. In third group, the average 5-year survival rate was 0.654 with the confidence interval of (0.584 and 0.723$)$. Analyzing by Meta regression didn't show any difference between 
groups (P-value $=0.732)$.

To assess the stability of the 5-year survival rate, sensitivity analysis was used. To do this, every single study was excluded from analysis and estimation of 5 -year survival rates was calculated (Table 2). The greatest impact was found in Ghavam Nasiri, by whose omission, the 5-year survival rates gets higher by 0.0081 . By removing the study of Mosavi, which had the largest sample size and consisted of approximately $30 \%$ of the whole participants, the 5-year survival rate is estimated to be greater by 0.0051 .

\section{Discussion}

With an analysis in the average age of onset of breast cancer in this study excluding the two study because there was no information regarding the average age of people; also in some analyses in which there was no reference to the average and diagnostic age of the disease, the Frequency Distribution Table for age distribution was available. We used this table to calculate the approximate mean and standard deviation of age. Moreover, in three studies in which the standard deviation age had not been mentioned, and only the minimum and maximum ages were referred to, by dividing the variety range by 6 , approximate estimation for standard deviation was obtained. Results of the analysis of onset of breast cancer homogeneous average age with the average age of 48.27 year-old with the confidence interval of (43.684 and 52.862) were resulted.

In a review study conducted on 85 articles, the incidence of disease in women were estimated as the 22 per 100,000 and its prevalence was 120 per 100,000 (Mousavi et al., 2007). In another study conducted in northwestern Iran the incidence was 28 per 100,000 (Taheri et al., 2012) which was high in comparison with that in developed countries, but low in comparison with that in developing countries (Ferlay et al., 2010).

Although the incidence of this cancer, in comparison with esophageal cancer, with an incidence of 180 per 100,000 people in Iran, is lower, since this disease is more common in the 40-60 years old (Tayebi et al., 2012), this is less than the incidence age of esophageal cancer in two decades. This means that more years of life are lost because of this cancer. Since the early stages of this cancer have a fair prognosis, education of women to be examined regularly and their awareness of the signs and symptoms of the disease are essential (Yavari \& Pourhoseingholi., 2007).

Survival rate of one, three and five years in our study was 95.6,80.8 and 69.5 respectively, which compared with survival rates of $91.6,70.2$ and 59.6, the percentage was higher in Jordan (Arkoob et al., 2010) but it was similar with survival rates of 94,83 and 74 percent in Vietnam (Lan et al., 2013).

As mentioned earlier, age is an independent risk factor for breast cancer (Jayasinghe et al., 2006). Screening in the international community, according to some studies, start at the age of 49, while the age of onset of breast cancer in our society starts a decade earlier than that in the international community (Sirous \& Ebrahimi., 2008).
Therefore, screening must begin at a younger age and in a more appropriate way. According to studies, the incidence of disease below age 35 causes a 2 -fold increase in the risk of mortality. Also, its incidence at age 70 and older increases the mortality rate (Querzoli et al., 2006).

Among different limitations of this study it should be noted that we only reviewed the articles that were written in Persian or English. Moreover, given that breast cancer is possible to occur in men, in most of the articles only women were taken into account, and in some articles a meager 5 percent and lower had included men. As mentioned before, it was not possible to categorize the data according to gender. Another limitation of this study is that in all studies, the survival rate has not been achieved by one single method and different methods such as KaplanMeier, the actuarial life table technique and Semi Markov Cox have been used to estimate the survival rates. Also, in some of these studies, the data have been collected personally while most of them had been obtained based on hospital information.

\section{References}

Akbari ME, Khayamzadeh M, Khoshnevis SJ, et al (2008). Five and ten years survival in breast cancer patients mastectomies vs. breast conserving surgeries personal experience. Iran $J$ Cancer Prev, 2, 53-6.

Akbari ME, Mirzaei HR, Soori H (2006). Five year survival of breast cancer in Shohada-e-Tajrish and Jorjani hospitals. Hakim Res J, 9, 39-44 [in Persian].

Arkoob K, Al-Nsour M, al-Nemry O, Al-Hajawi B (2010). Epidemiology of breast cancer in women in Jordan: patient characteristics and survival analysis. East Mediterr Health $J, \mathbf{6}, 1032-8$.

Babaei GR, Feizi A, Keshavarz M (2005). Assessment of different method of treatment and surgical effect on survival rate in patients with breast cancer. Daneshvar, 13, 19-28 [in Persian].

Fallahzadeh H, Momayyezi M, Akhundzardeini R, et al (2014). Five year survival of women with breast cancer in Yazd. Asian Pac J Cancer Prev, 15, 6597-601.

Fardmal J, Mafi M, Sadighi-Pashaki A (2014). Factors affecting survival in breast cancer patients referred to the Darol Aitam-e Mahdieh center. J Zanjan Univ Med Sci, 22, 10515 [in Persian].

Fazeli Z, Najafian-Zade M, Eshrati B, et al (2014). A five-year evaluation of epidemiological, geographical distribution and survival analysis of breast cancer in Markazi Province, 2007-11. Arak Medical University J, 16, $72-9$ [in Persian].

Ferlay J, Shin HR, Bary F, et al (2010). Cancer incidence and mortality worldwide, international agency for research on cancer, GLOBOCAN 2008 v1.2, Lyon.

Fouladi N, Amani F, Harghi AS, et al (2011) Five year survival of women with breast cancer in ardabil, north-west of Iran. Asian Pac J Cancer Prev, 12, 1799-801.

Ghavam-Nasiri MR, Anvari K, Nowferesti GH, et al, (2005). Locally advanced breast cancer: an experience in Mashhad, north-east of Iran, 1995-1999. Arch Iranian Med, 8, 206-10.

Gohari MR, Moghadamifard Z, Abolghasemi J, et al (2013). Prognostic factors of metastases in breast cancer patients using the recurrent andersen-gill model. Koomesh, 14, 483-9 [in Persian]

Hagigat S, Olfat Bakhsh O, Sajadian A, et al (2013). Survival rate and its correlated factors in breast cancer patients referred to Breast Cancer Research Center. Iran J Breast Dis, 6, 
28-36 [in Persian].

Harirchi I, Karbakhsh M, Kashefi A (2004). Breast cancer in Iran: results of a multi-center study. Asian Pac J Cancer Prev, 5, 24-7.

Hasanzadeh S (2014). Estimate of survival rate in patient with breast cancer in Fasa between 2002-2012. Thesis [in Persian].

Heydari ST, Mehrabani D, Tabei SZ, et al (2009). Survival of breast cancer in Southern Iran. Iran J Cancer Prev, 2, 51-4.

Islamic Republic of Iran. Ministry of Health and Medical Education. Center for Disease Control \& Prevention. Noncommunicable Deputy. Cancer Office (2009). Iranian Annual of National Cancer Registration Report.

Jayasinghe UW, Tylor R, Boyages J, (2005). Is age at diagnosis an independent prognostic factor for survival following breast cancer? ANZ J Surg, 75, 762-7.

Karimi A, Delpisheh A, Sayehmiri K, et al (2014). Predictive factors of survival time of breast cancer in Kurdistan province of Iran between 2006-2014: a Cox regression approach. Asian Pac J Cancer Prev, 15, 8483-8.

Kolahdoozan S, Sadjadi AR, Radmard AR, et al (2010). Five Common Cancers in Iran. Arch Iran Med, 13, 143-6.

Lan NH, Laohasiriwong W, Stewart JF (2013). Survival probability and prognostic factors for breast cancer patients in Vietnam. Glob Health Action, 6, 1-9.

Miller BA, Feuer EJ, Hankey BF (2004). Recent incidence trends for breast cancer in women and the relevance of early detection: an update. CA Cancer J Clin, 43, 27-30.

Moetamed N, Negin H, Taleei AR (2004). Evaluation of risk factor on women of older than 35 years with breast cancer in Shiraz, 2001.J Zanjan Univ Med Sci, 12, 25-33 [in Persian].

Mokhtari-Hesari P, Pasha E, Gohari MR (2013). Prognostic Factors on Metastasis and Mortality Incidence in Breast cancer with disease and mortality Model. Iran J Breast Dis, 6, 36-42 [in Persian].

Mokarian F, Mokarian Sh, Ramezani A (2013). Relations of disease-free survival and overall survival with age and primary metastases in patients with breast cancer. $J$ Isfahan Univ Med Sci, 31, 112-20 [in Persian].

Moradi-Marjaneh M, Homaee-Shandiz F, Shamsian SAA, et al (2005). Association between Her2/neu, P53, ER and Pr genes with survival rate in Iranian women with breast cancer. Iran J Breast Dis, 1, 7-17. [Persian]

Mousavi SM, Mohagheghi MA, Mousavi-Jerrahi A, et al (2008). Outcome of Breast Cancer in Iran: A Study of Tehran Cancer Registry Data. Asian Pac J Cancer Prev, 9, 275-8.

Mousavi SM, Montazeri A, Mohagheghi MA, et al (2007). Breast cancer in iran: an epidemiological review. Breast $J$, 13, 383-91.

Movahedi M, Haghighat SH, Khayamzadeh M, et al (2012). Survival rate of breast cancer based on geographical variation in Iran, a national study. Iran Red Creacent Med $J, \mathbf{1 4}, 798-804$

Querzoli P, Pedriali M, Rinaldi R, et al (2006). Axillary Lymph node nonmetastases are prognostic relapse in breast cancer patient. Clin Cancer Res, 12, 6696-701.

Rajaeifard A, Talei A, Baneshi M (2006). Survival analysis models for breast cancer patients in Shiraz, 1993-2002. J Med Res, 3, 41-50 [in Persian].

Rezaianzadeh A, Peacock J, Reidpath D, et al, (2009). Survival analysis of 1148 women diagnosed with breast cancer in Southern Iran. BMC Cancer, 9, 168.

Salehi M, Gohari M, Vahabi N, et al (2013). Comparison of artificial neural networks and cox regression models in survival prediction of breast cancer patients. J Ilam Univ Med Sci, 21, 120-8 [in Persian].

Sharifian A, Pourhoseingholi MA, Emadedin M, et al (2015).
Survival Rate of Breast Cancer in Iranian Patients: a Meta-Analysis burden of breast cancer in Iranian women is increasing. Asian Pac J Cancer Prev, 16, 5049-52.

Sirous M,Ebrahimi A (2008). The epidemiology of breast masses among women in Isfahan. Iran J Surg, 16, 51-6.

Taghavi A, Fazeli Z, Vahedi M, et al (2012). Increased trend of breast cancer mortality in Iran. Asian Pac J Cancer Prev, 13, 367-70.

Taheri NS, Bakhshandehnosrat S, Aarabi M (2012). Epidemiological pattern of breast cancer in Iranian women - is there an ethnic disparity?. Asian Pac J Cancer Prev, 13, 4517-20.

Tayebi M, Shabestani-Monfared A, Moslemi D (2012). A 10 year survey of cancer in patients who referred to Shahid Rajai Radiotherapy Center in North of Iran (2000-2009). J Babol Univ Med Sci, 14, 97-101 [in Persian].

Vahdaninia M, Harirchi I, Montazeri (2002). Assessment of five year survival rate in breast cancer women referred to the Emam Khomeini Hospital: A Prospective study. Payesh, 2, 141-8 [in Persian].

Vostakolaei FA, Broeders MJ, Rostami N, et al (2012). Age at Diagnosis and Breast Cancer Survival in Iran. Int J Breast Cancer, 2012, 517976.

World health organization regional Office for the Eastern Mediterranean (2006). Guidelines for the management of Breast Cancer, EMRO Technical Publications Series 31, Cairo.

Yaghmaei S, Bani-Hashemi G, Ghorbani R (2008). Survival rate following treatment of primary breast cancer in Semnan, Iran (1991-2002). J Semnan Univ Med Sci, 9, 111-7 [in Persian].

Yavari P, Pourhoseingholi MA (2007). Socioeconomic factors association with knowledge and practice of breast selfexamination among Iranian women. Asian Pac J Cancer Prev, 8, 618-22.

Ziaei JE, Sanaat Z, Asvadi I, et al (2013). Survival analysis of breast cancer patients in Northwest Iran. Asian Pac J Cancer Prev, 14, 39-42. 